\title{
Variable Launch System for the Metrology of EOCBs
}

\section{Robert Ferguson*, Irshaad Fatadin and Ka-ming Liu}

\author{
National Physical Laboratory, London, United Kingdom
}

\begin{abstract}
Optical links within data centres are critical components supporting increasing data consumption. These links vary in technologies, and metrological standards for the industry are still being developed. Concerning specific multimode interconnects for high data rates, we present the use of a system incorporating a variable controlled launch condition as a platform for characterizing the effect of temperature on electro-optical circuit boards (EOCBs) for metrology. We examine the performance impact on key parameters such as bit error ratio (BER). We conclude with a vision on the future of data centre networks and the requirement for standard bodies to introduce new industry-agreed metrology techniques for standardization.
\end{abstract}

\section{Keywords}

Optical metrology, EOCB, Optical interconnect

\section{Introduction}

Proliferation of "big data" in business and exponentially rising internet usage has led to a rapid growth in data traffic globally. Unprecedented demand for data centres and high-speed data transmission has driven the adoption of short-range optical links in data cabinets, links that have been primarily electrical for the past century. Integrated photonic-based solutions have emerged, and one key technology is the creation of the electrooptical circuit board (EOCB). Widespread adoption of EOCBs will be essential to keeping up with data requirements and we expect optical "intrarack" links to become dominant features within hyperscale data centres [1].

EOCBs offer greatly improved data transfer speeds (>1000 $\mathrm{Gb} / \mathrm{s} / \mathrm{m})$ compared to copperbased counterparts ( $100 \mathrm{~Gb} / \mathrm{s} / \mathrm{m})$. Broadly, there are three interconnect technologies - fiber-optic laminate, polymer waveguides, and planar glass waveguides [2-4]. As EOCB technology reaches maturity, there is a pressing need to develop standards for the industry. Recent work within IEC technical committees (TC86/JWG9) has led to the adoption of a reliable measurement definition system for optical interconnects - a vital prerequisite for future standardization [5]. This includes the standardizations of EOCB performance characterisation which includes understanding the impact of local environmental conditions on their efficiency.

\section{Materials and Methods}

At NPL we simulated the thermal effects on

*Corresponding author: Robert Ferguson, National Physical Laboratory, London, United Kingdom Accepted: August 12, 2021; Published: August 14, 2021

Copyright: (C) 2021 Ferguson R, et al. This is an open-access article distributed under the terms of the Creative Commons Attribution License, which permits unrestricted use, distribution, and reproduction in any medium, provided the original author and source are credited.

Ferguson et al. Int J Opt Photonic Eng 2021, 6:037

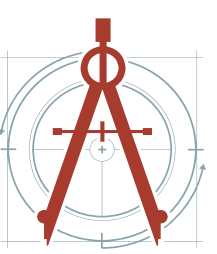


EOCBs using a localised heat source - this would be comparable to hot spots generated by other on-board components such as CPU's, transceivers, etc. developing inside the data cabinets where these boards would be used. We targeted the photolithographic polymer waveguides on an EOCB with a thermal load and analyzed the effects this would have on bit error ratio (BER), attenuation and encircled flux (EF).

The experimental setup consists of a modified NPL Variable Launch System [6,7] and a commercial unit capable of applying controlled thermal loads to targeted areas of the circuit board. The experimental setup was capable of applying temperatures up to $225{ }^{\circ} \mathrm{C}$, however, testing was limited to $-5^{\circ} \mathrm{C}$ to 80 ${ }^{\circ} \mathrm{C}$ which was determined to cover the practical conditions that these boards should be subjected to within a cabinet environment. Throughout the measurements the room environment was monitored, with ambient temperature controlled to $22{ }^{\circ} \mathrm{C}$. Ambient board temperature was monitored by a K-type thermocouple.

The variable launch system acted as a flexible platform for exploring performance of various optical waveguides across the entire EOCB. The system allowed for the user to position $850 \mathrm{~nm}$ spots of variable size and numerical aperture (NA) onto the front facet of an optical waveguide and then image the resulting output, using a CCD.

As seen in Figure 1 the system utilized an 850 nm Vertical Cavity Surface Emitting Laser (VCSEL) source, an optical launch board that produced the required launch spot (comprising components from L1 to L4), the optical circuit board and an optical receive board to measure the output (components L8 to CCD2). The system itself was mounted onto an optical bench while under test to minimize vibrational effects. The fiber-coupled VCSEL was mounted with a collimating objective and a computer-controlled filter wheel allows for light to be blocked when necessary. A position for placing fixed neutral density filters in the collimated path to control light intensity is also present. A second objective then focuses the light into the fiber. The delivery optical fiber $F$ is also equipped with a shaker to provide speckle/phase scrambling over the averaging time of the measurements.

For characterisation of the launch in terms of $\mathrm{EF}$, a commercial "Modal Explorer" was integrated into the system and used to assess the radial

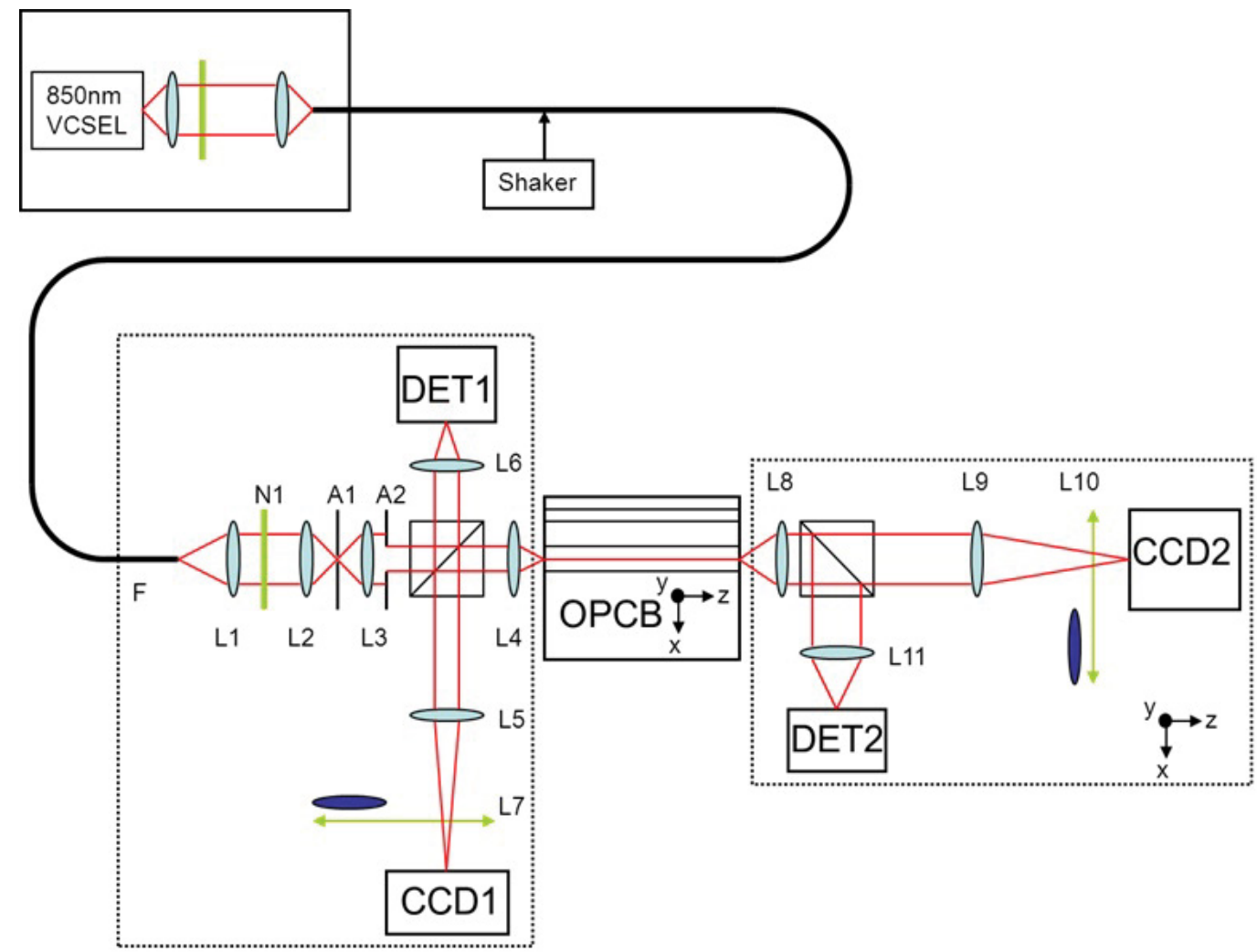

Figure 1: Schematic of the NPL Variable Launch System. 

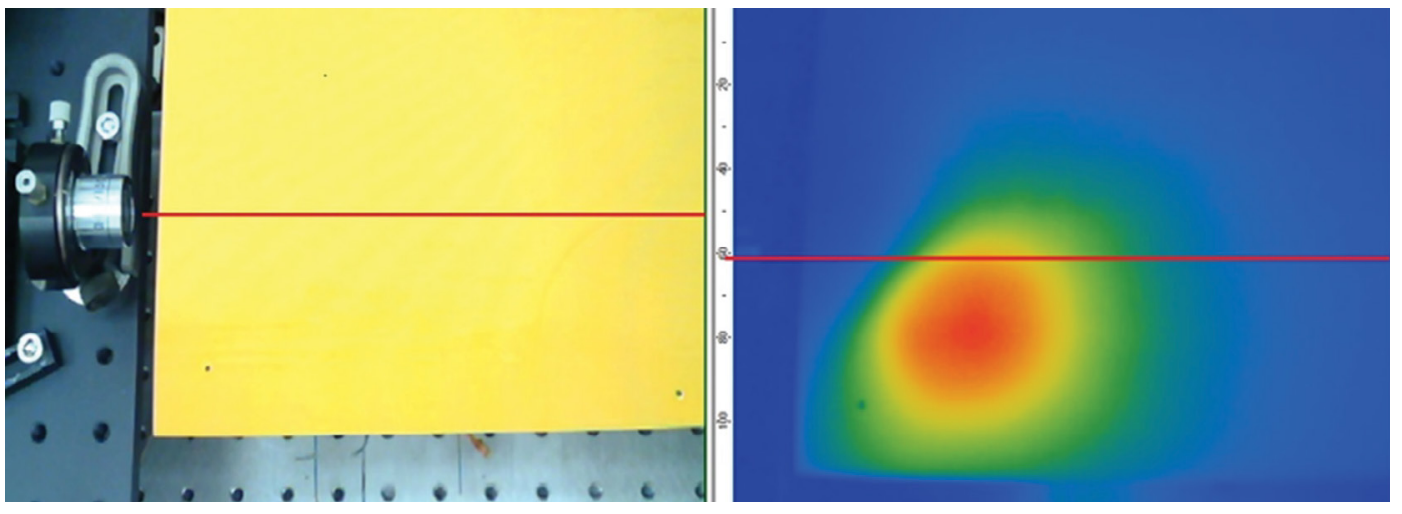

Figure 2: Example thermal hotspot on an EOCB. The red line marks a waveguide group axis.

energy distribution of the launch signal. Modal conditioning was also incorporated into the system at the launch fiber to ensure it conformed to current recommended launch profiles as designed in IEC 62496-2:2017 (E). For a certain launch profile, the light would pass into $5 \mathrm{~m}$ of graded-index multimode fiber (GI-MMF), wrapped 20 times around a $38 \mathrm{~mm}$ diameter mandrel. The output of this mandrel would then be passed through a mode conditioner to produce the required modefiltered optical intensity profile, complying with EF requirement IEC 61290-4-1.

For optical interconnects, understanding the way the light enters the waveguide is vital in ensuring minimal loss and thus maximize efficiency. The variable launch system platform allows for careful control over the launch conditions enabling experimental measurements for the performance for a large range of launch conditions. The relative ease of modification, such as for ensuring EF compliance, allows for experiments to be done while conforming to a range of criteria specified within current standards $[8,9]$.

The final setup (accommodating for modal conditioning and applied thermal loads) allowed for controlled launch conditions through test waveguides to be observed through an infrared CCD across a range of temperature conditions. This can be seen in Figure 2, as we apply the controlled thermal load across a particular waveguide group axis. The signals were then analyzed to assess the impact the thermal load had for different parameters. Total power was assessed by summing all the pixels found within a given power intensity area and comparing. Total attenuation was calculated by comparing the total input and output of the target waveguide, accounting for expected intrinsic waveguide and coupling losses. Encircled flux was assessed using the modal explorer to receive the final output. Bit error ratio was examined through launching intensity-modulated signals through the EOCB waveguide and analyzing the result through a real-time oscilloscope sampling at $20 \mathrm{G}$ Samples/s with the transmitted signal recovered through a photo-receiver. An arbitrary waveform generator was used to generate a pseudo-random binary sequences electrical signal in this instance, driving an SFP+ transceiver module that was then launched into the nominated waveguides.

\section{Results and Discussion}

We investigated the effect of the thermal load using the launch system on the parameters of total attenuation, encircled flux, and bit error ratio.

We present our findings for WG7 in Figure 3, where we load had a measurable effect on the total attenuation across the waveguide of the EOCB. The maximum range of measured total attenuation was $\sim 0.3 \mathrm{~dB}$. The expanded uncertainties associated with the measurements are estimated as ranging from U95 <+/- $0.1 \mathrm{~dB} @ 20^{\circ} \mathrm{C}$ and +/- $0.20 \mathrm{~dB} @$ $80^{\circ} \mathrm{C}$. These are derived from observed repeatable measurements and the established system uncertainties [6]. This loss could have a noticeable effect on bandwidth efficiencies, especially once we consider the increasing complexity of these boards.

We provided characterisation for EF, defined as a fraction of the cumulative near-field power to the total output power as a function of radial distance from the optical centre. An observable effect was found as we increased the thermal load, as seen in Figure 4. This is consistent with that of higher-order modes being decoupled from the waveguide, due to changes in the core-cladding refractive index 


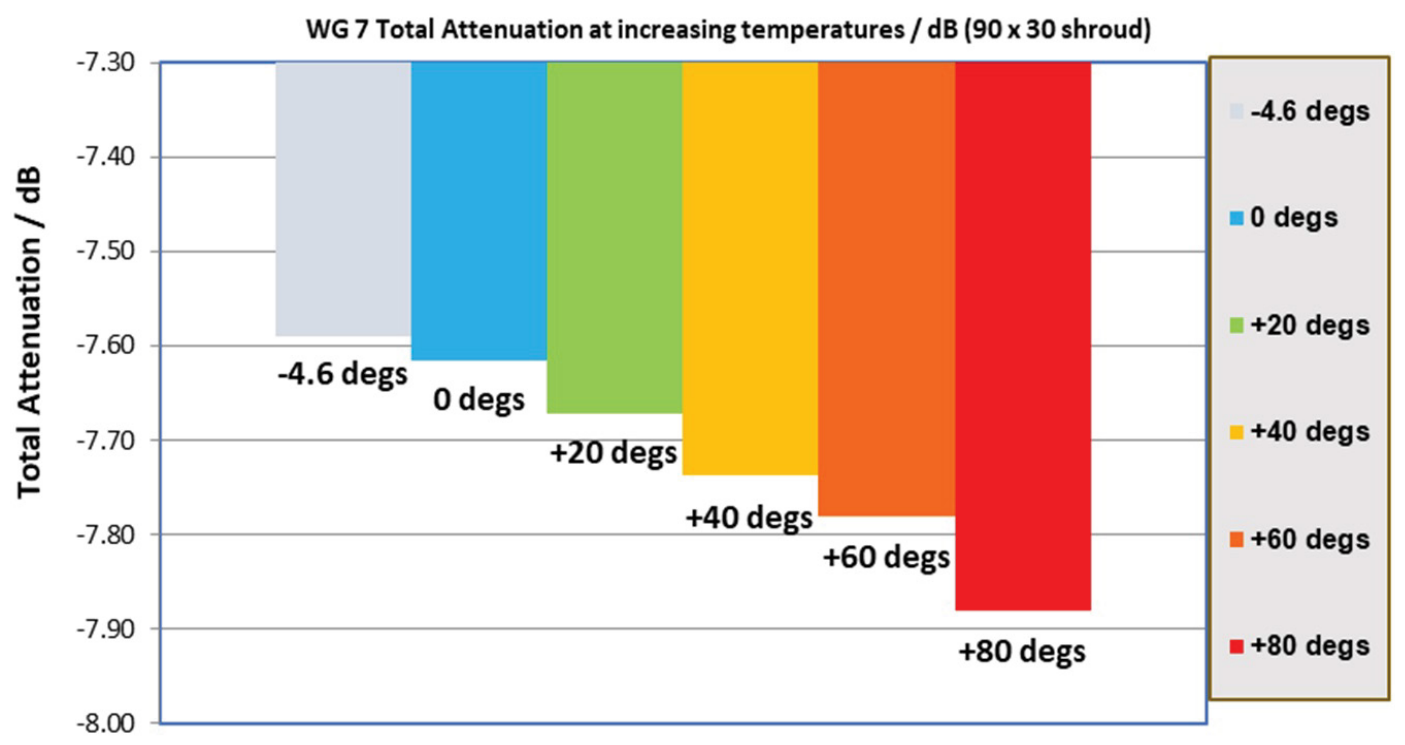

Figure 3: Total attenuation against temperature for different waveguides.

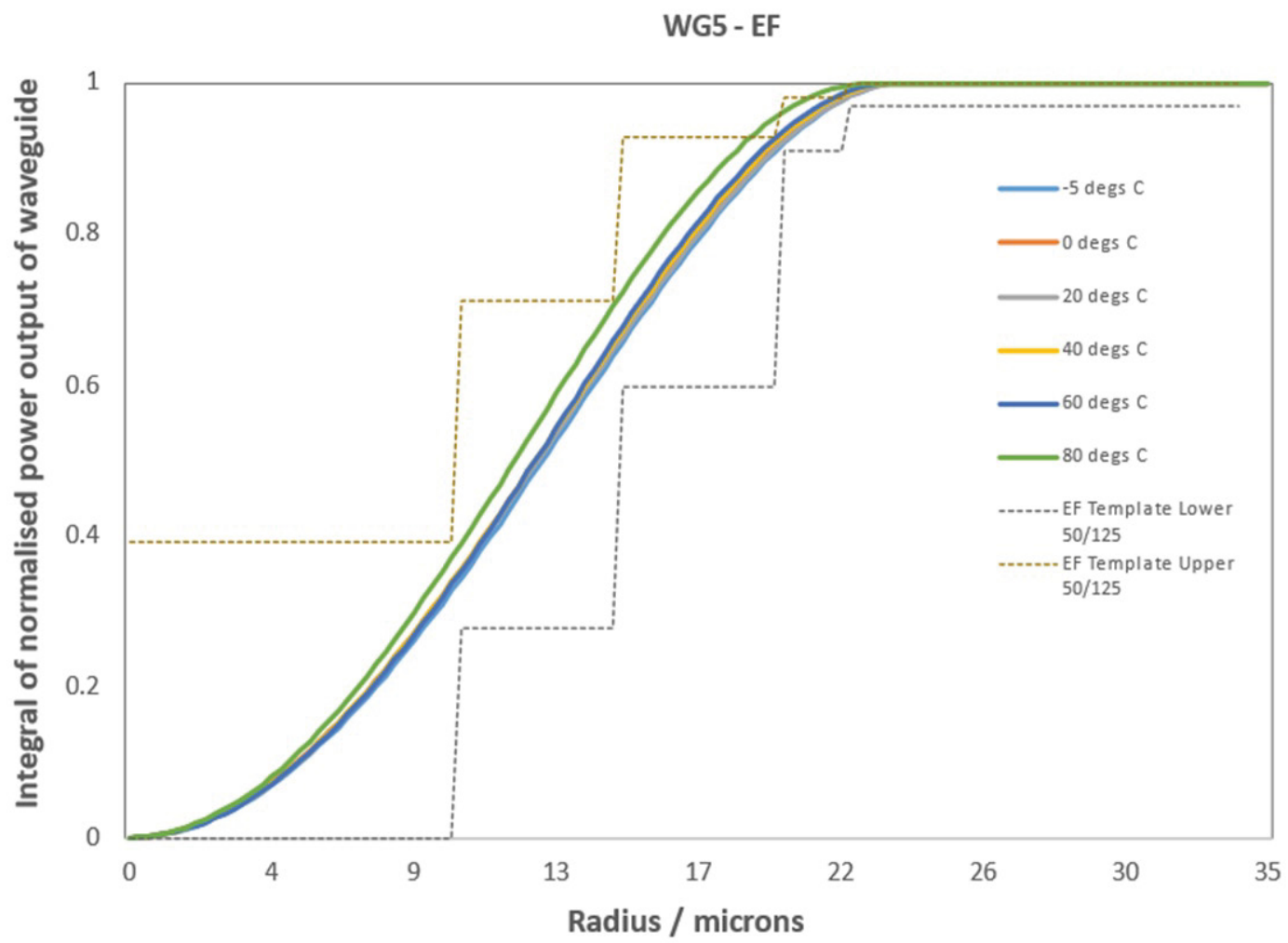

Figure 4: Normalized power output and waveguide radius.

ratio. We found that under exceptional thermal stress that the resulting performance shift exceeds that of templates recommended by current IEC standards. This can be seen at $80^{\circ} \mathrm{C}$ in Figure 4 as it breaches the upper limit of the EF template.

Finally, we investigated how BER was affected by the application of thermal loads. The intensitymodulated signal was launched onto two separate waveguides on the same board. Similar to the EF measurements, the launch configuration utilized a modal conditioner. The modal profile generated complied with restricted launch EF requirements IEC 61300-1/IEC 61280-4-1. Our findings for the two waveguides are shown in Figure 5 . We found that BER rose with increased thermal stress, consistent with performance expectations based on the results 


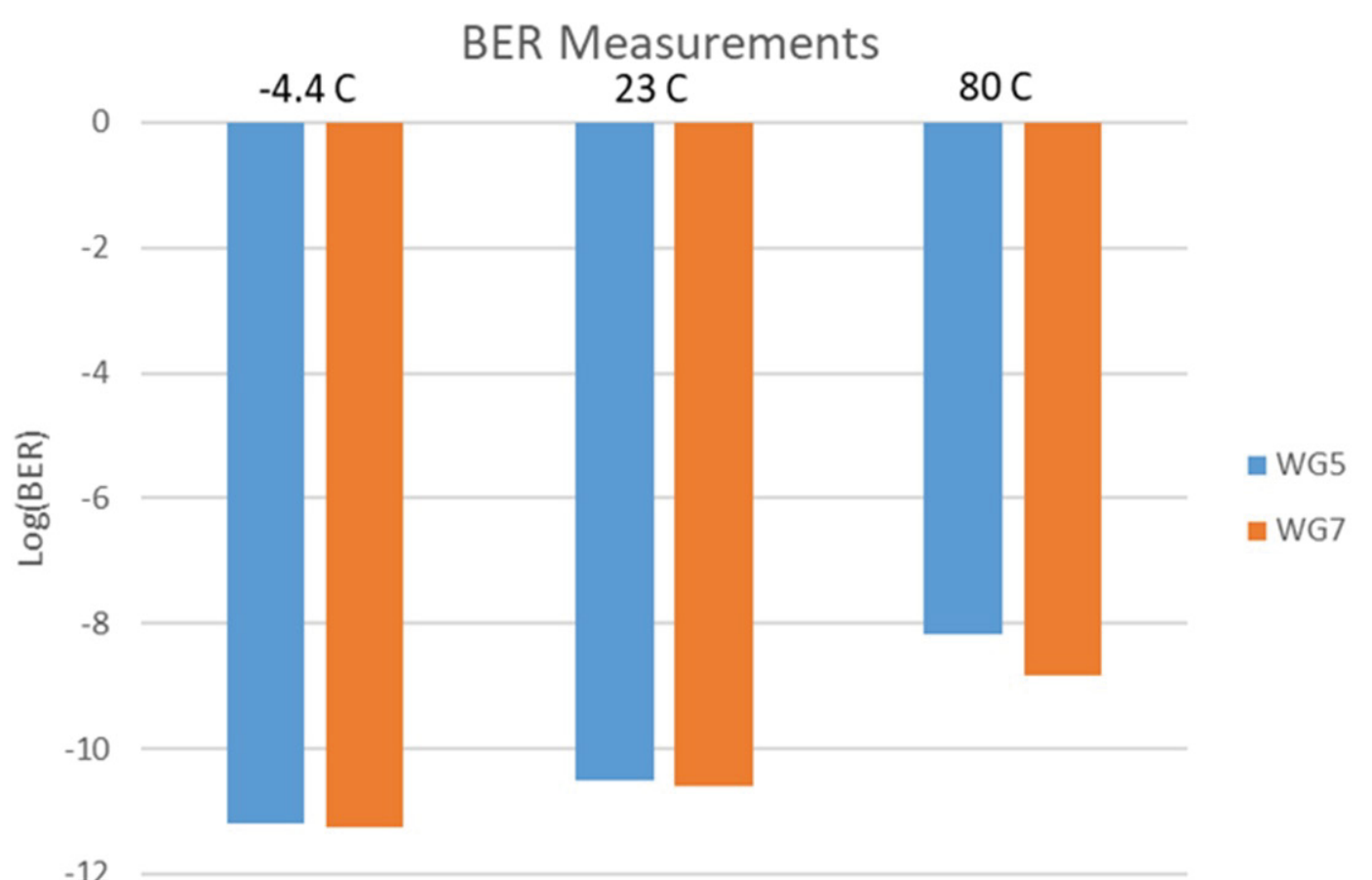

Figure 5: Bit error ratio at three temperatures, for two waveguides.

for total attenuation and the shift in EF profiles. Signal degradation was observed for the transmitted data at the extreme temperature of $80{ }^{\circ} \mathrm{C}$ for both waveguides. As BER is a particularly critical parameter and a key metric for data channel performance and has been previously characterised at room temperature [10], this work highlighted the significant impact localised temperature variation can have for EOCB utilization. Management of heat alongside carefully controlled launch conditions as prescribed by practical metrology standards is needed then to maintain industry-required performance.

\section{Conclusions}

The variable launch system presented acted as a robust platform on which to perform metrology on EOCBs and allowed us to perform experimental simulations of EOCB performance with regards to temperature conditions within a data cabinet. We demonstrated that the heating of the boards can have noticeable effects on their functional performance, potentially compromising existing standards. Key future work investigating the impact of the measured attenuation on the changes in BER performance for different modulation formats such 4-PAM, as well as the associated uncertainty contributions will need to be carried out.

As noted in the IEC standard - "Independent repeatability of waveguide measurements is still very difficult to achieve due to the lack of clarity on how measurement conditions are specified..." There is significant progress needed before standards can properly support the rapid development of these pluggable optical interconnects within the data industry. Good understanding of the measurement conditions must accompany the increasing applications of EOCBs to keep up with the demand of increasing data transfer rates. While significant work from industrial and academic institutions have been carried out in assessing passive boards and photonic interconnects $[3,11]$, there still exists wide latitude in optical circuit board design and operating conditions. There is a need to establish reliable metrology techniques then, such as using systems such as a variable launch system, in order to ensure measurement harmonization for the global industry [12].

\section{Acknowledgments}

This project 19SIP05 TTPWC has received funding from the EMPIR programme co-financed by the Participating States and from the European Union's Horizon 2020 research and innovation programme, Funder ID: 10.13039/ 100014132.

\section{References}

1. Senko (2020) Data centre network: Impact on optical 
interconnect \& component technology. Lightwave Broadcast.

2. Ingham JD, Bamiedakis $\mathrm{N}$, Penty RV, White $\mathrm{IH}$, DeGroot JV, et al. (2006) Multimode siloxane polymer waveguides for robust high-speed interconnects. Conference on Lasers and Electro-Optics and 2006 Quantum Electronics and Laser Science Conference, IEEE, Long Beach, CA, USA.

3. Pitwon RCA, Wang $K$, Jones JG, Papakonstantinou I, Baghsiahi H, et al. (2012) First light: Pluggable optical interconnect technologies for polymeric electro optical printed circuit boards in data centers. Journal of Lightwave Technology 30: 3316-3329.

4. Su K, DeGroot JV, Ann W Norris AW Jr, Lo PY (2005) Siloxane materials for optical applications. Advanced technologies and venture business, Dow corning corporation, 2200 W. SPIE, Salzburg Road, Midland, MI, USA 6029: 48686.

5. IEC (2017) Optical circuit boards - Basic test and measurement procedures - Part 2: General guidance for definition of measurement conditions for optical characteristics of optical circuit boards.

6. Ives D, Ferguson R, Harris S (2011) Development of a variable launch attenuation and isolation measurement system for optical waveguides. Appl Opt 50: 4268-4275.

7. Ferguson R, Fatadin I, Liu KM, Barbeito I, Hart C, et al. (2021) Metrology and standardization of high-speed pluggable optical interconnects. PHOTOPTICS, 63-67.

8. IEC (2016) Fibre optic interconnecting devices and passive components - Basic test and measurement procedures - Part 1: General and guidance.

9. IEC (2020) Fibre optics - Multimode launch conditions - Part 1: Launch condition requirements for measuring multimode attenuation.

10. Bamiedakis N, Hashim R, Penty RV, White IH (2012) Regenerative polymeric bus architecture for boardlevel optical interconnects. Opt Express 20: 1162511636.

11.Pitwon $R$ (2016) Advances in photonic interconnect for data centre subsystems. $4^{\text {th }}$ Symposium on Optical Interconnect in Data Centres, ECOC.

12.Selviah DR, Walker AC, Hutt DA, Wang K, McCarthy $A$, et al. (2010) Integrated optical and electronic interconnect PCB manufacturing research. Circuit World 36: 5-19. 